\title{
Interventions for promoting information and communication technologies adoption in healthcare professionals (Protocol)
}

Gagnon MP, Légaré F, Labrecque M, Frémont P, Pluye P, Gagnon J, Gravel K

Gagnon MP, Légaré F, Labrecque M, Frémont P, Pluye P, Gagnon J, Gravel K.

Interventions for promoting information and communication technologies adoption in healthcare professionals.

Cochrane Database of Systematic Reviews 2006, Issue 3. Art. No.: CD006093.

DOI: 10.1002/14651858.CD006093.

www.cochranelibrary.com 
TABLE OF CONTENTS

HEADER 1

ABSTRACT

BACKGROUND

OBJECTIVES

METHODS

REFERENCES

WHAT'S NEW

CONTRIBUTIONS OF AUTHORS

DECLARATIONS OF INTEREST

INDEX TERMS 
[Intervention Protocol]

\section{Interventions for promoting information and communication technologies adoption in healthcare professionals}

Marie-Pierre Gagnon ${ }^{1}$, France Légaré2 ${ }^{2}$ Michel Labrecque ${ }^{3}$, Pierre Frémont ${ }^{4}$, Pierre Pluye ${ }^{5}$, Johanne Gagnon ${ }^{6}$, Karine Gravel ${ }^{7}$

1Unité de recherche évaluative, Centre hospitalier universitaire de Québec - Hôpital St-François d'Assise, Québec, Canada. 2 Research Center, CHUQ, St-François D'Assise Hospital, Québec, Canada. ${ }^{3}$ Département de médecine familiale, Centre hospitalier universitaire de Québec - Hôpital St-François d'Assise, Québec, Canada. ${ }^{4}$ Centre Hospitalier de l'Université Laval, Québec, Canada. ${ }^{5}$ Département de médecine familiale, Université McGill, Montréal, Canada. ${ }^{6}$ Faculté des sciences infirmières, Université Laval , Québec, Canada. ${ }^{7} \mathrm{CHUQ}$, St-François D'Assise Hospital, Québec, Canada

Contact address: Marie-Pierre Gagnon, Unité de recherche évaluative, Centre hospitalier universitaire de Québec - Hôpital St-François d'Assise, 10, rue de l'Espinay, D1-724, Québec, Québec, G1L 3L5, Canada. Marie-Pierre.Gagnon@mfa.ulaval.ca.

Editorial group: Cochrane Effective Practice and Organisation of Care Group.

Publication status and date: Edited (no change to conclusions), published in Issue 4, 2008.

Citation: Gagnon MP, Légaré F, Labrecque M, Frémont P, Pluye P, Gagnon J, Gravel K. Interventions for promoting information and communication technologies adoption in healthcare professionals. Cochrane Database of Systematic Reviews 2006, Issue 3. Art. No.: CD006093. DOI: 10.1002/14651858.CD006093.

Copyright @ 2008 The Cochrane Collaboration. Published by John Wiley \& Sons, Ltd.

\section{A B S T R A C T}

This is a protocol for a Cochrane Review (Intervention). The objectives are as follows:

To carry out a systematic review of the effects of interventions to promote the integration of ICT applications into health care professionals' practices.

Five comparisons are planned:

(1) Single intervention specifically designed to promote ICT adoption in health care settings compared to no intervention.

(2) Single intervention specifically designed to promote ICT adoption in health care settings compared to any other intervention.

(3) Multifaceted interventions specifically designed to promote ICT adoption in health care settings compared to no intervention.

(4) Multifaceted interventions specifically designed to promote ICT adoption in health care settings compared to single intervention designed to promote ICT adoption in health care settings.

(5) Multifaceted interventions specifically designed to promote ICT adoption in health care settings compared to any other multifaceted intervention. 


\section{B A C K G R O U N D}

Information and communication technologies (ICTS) have the potential to address many of the challenges that health care systems are currently confronting. Globally, "ICT encompasses all those digital and analogue technologies that facilitate the capturing, processing, storage and exchange of information via electronic communication" (Health Canada 2006). The expression e-health is also increasingly used to refer to ICT applications in the health care domain (Oh 2005; Eysenbach 2001). Thus, this review considers health care ICT and e-health applications as equivalent.

The following classification identifies the five broad categories of e-health applications (Open Clinical 2006):

1. Electronic Medical Records (including patient records, clinical administration systems, digital imaging \& archiving systems, eprescribing, e-booking);

2. Telemedicine and telecare services;

3. Health information networks;

4. Decision support tools for health care professionals;

5. Internet-based technologies and services.

ICT applications have the potential to improve information management, access to health services, quality of care, continuity of services, and costs containment (Gov. Canada 1999). Even if the efficacy of some of these technologies, such as teleconsultations (Currell 2000), computerised health records (Erstad 2003), and clinical information-retrieval technologies (Pluye 2005) is not proven yet, other ICTs, such as some decision-support systems (Garg 2005; Kawamoto 2005) and computerised reminders (Bennett 2003), have shown benefits for the health care system and may improve patient health outcomes. Furthermore, with increasing computerization in every sector of activity, ICTs are likely to become tools that are part of health care professional practice. Nonetheless, it appears that ICTs such as electronic medical records and Internet remain underused by health care professionals (Berner 2005; Brooks 2006). Human and organisational factors have been identified as the main causes of ICT implementation failure (Aarts 2004; Lorenzi 1997).

The optimal integration of ICTs into health care professionals' practices should be based upon the highest level of scientific evidence available with respect to implementation strategies (Grimshaw 2004; Moehr 2000). Previous studies have found that training is a major determinant of ICT adoption by health care professionals and also influences the integration of these technologies into clinical practices (Allen 2000; Knonick 2003). However, many factors influence the effectiveness of educational strategies, such as the characteristics of the learner, the intervention itself, the characteristics of the behaviour that the intervention is trying to change, and the context in which the intervention is conducted (Farmer 2003).

Characteristics of the intervention include the source of the information, the content and the channel by which it is delivered (Marriott 2000; Kanouse 1995). The credibility of the information source is based upon several aspects, such as expertise and knowledge, trustworthiness, and message attributes (Tseng 1999; Wathen 2002). Research on the specific characteristics of successful interventions to promote ICT adoption into clinical practice is limited. However, other studies on effective strategies to change clinical practice indicate that training interventions need to be sufficiently persuasive, informative and relevant to the learner (Davis 1999). Personalised feedback and/or tailored messages may also be more useful for changing behaviour than non-tailored messages (Kreuter 2001; Kreuter 1996; Bull 2001).

With respect to characteristics of the behaviour that is targeted by the intervention, systematic reviews on the adoption of clinical practice guidelines found that behaviour change is facilitated when they are simple, compatible with physicians' norms and values and do not require acquisition of new knowledge or skills (Burgers 2003; Grilli 1994; Grol 1998). Moreover, adoption of clinical guidelines by physicians would be facilitated by the perception of their concordance with previous experiences as well as their confidence in the source of the information (Davis 1999; Slotnick 1999).

The characteristics of professional groups, organisations and context also influence the success of an intervention. At the group level, the introduction of ICT can bring modifications to roles and responsibilities of health care professionals, which represents a potential source of resistance to adoption (Markus 1983). Likewise, conflicts can be present with respect to the delimitation of professional boundaries (Massaro 1993; McLaughlin 1998). Furthermore, structural and contextual characteristics of health care organisations can influence the integration of ICTs into clinical practices (Kimberly 1981). Factors such as hospital location and size, participation of professionals into decision-making and management support have been found to impact on ICT adoption (Carman 1996; Gagnon 2005; Lapointe 1999).

In summary, the effectiveness of interventions aiming at the integration of ICT applications into health care professionals' practices are likely to be influenced by various factors pertaining to individual, group, organisational, and contextual characteristics and by the very nature of the intervention (Grol 2004). Furthermore, interrelations between these factors are important to consider since they can influence how scientific evidence is integrated into practices (Street 1997).

\section{O B JECTIVES}

To carry out a systematic review of the effects of interventions to promote the integration of ICT applications into health care professionals' practices.

Five comparisons are planned:

(1) Single intervention specifically designed to promote ICT adoption in health care settings compared to no intervention.

(2) Single intervention specifically designed to promote ICT adoption in health care settings compared to any other intervention.

(3) Multifaceted interventions specifically designed to promote ICT adoption in health care settings compared to no intervention.

(4) Multifaceted interventions specifically designed to promote ICT adoption in health care settings compared to single intervention designed to promote ICT adoption in health care settings.

(5) Multifaceted interventions specifically designed to promote ICT adoption in health care settings compared to any other multifaceted intervention. 


\section{METHODS}

\section{Criteria for considering studies for this review}

\section{Types of studies}

The following study designs will be considered: randomised controlled trials (RCTs); controlled clinical trials (CCTs); controlled before and after studies (CBAs); and interrupted time series analyses (ITS) where there is a clearly defined point in time when the intervention occurred and at least three data points before and three after the intervention (Ramsay 2003). Studies published in all languages will be included.

\section{Types of participants}

Any health care professionals, including professionals in training when they are responsible for patient care (residents, fellows, and other registered health professionals), provided with an intervention aimed at promoting their adoption or the use of any type of ICTs, or both, in their practice.

\section{Types of interventions}

Interventions promoting the adoption and use of any type of ICTs (telehealth, computers, e-mail, Internet, electronic databases, decision-support systems, electronic prescription systems, computerised reminders, electronic health records, microprocessor health cards, etc.) will be included.

The intervention has to go beyond the provision of or access to an ICT application; i.e., there should be a planned strategy to promote adoption of the ICT application.

\section{Types of outcome measures}

There are two types of outcome measures that will be of interest in this review. First, the primary outcomes of the interventions considered will mainly concern the adoption or use of the ICT application or both (e.g., the number of teleconsultations conducted by a physician via videoconference, the number of tests ordered electronically). These outcomes are important to consider in the present review since they directly influence process outcomes, and, ultimately, health and cost outcomes.

Second, we will consider any objective measure of clinical performance or process outcome (e.g., number of tests ordered or decision to prescribe a particular drug), or patient health outcomes (such as blood pressure, length of hospital stay, etc.). Measures of health practitioners' knowledge, attitudes or satisfaction will be included in this review as they may provide useful secondary information.

Studies only reporting knowledge, attitudes or satisfaction, with no objective measure of process or patient outcomes, will not be included in the systematic review on the effectiveness of interventions. However, these studies will be considered in a complementary systematic review of barriers and facilitators to ICT adoption that will be conducted simultaneously.

\section{Search methods for identification of studies}

Specific strategies defined with the help of an information specialist will be used to search the EPOC register (See SPECIALISED REGISTER under GROUP DETAILS) and the database of studies awaiting assessment. Additional searches will be conducted on MEDLINE and the following electronic databases will be searched: (a) The EPOC Register (and the database of studies awaiting assessment) was reviewed (see SPECIALISED REGISTER under GROUP DETAILS);

(b) The Cochrane Central Register of Controlled Trials (CENTRAL) and the Database of Abstracts of Reviews of Effectiveness;

(c) Additional searches will be conducted on MEDLINE and all relevant databases.

Search strategies for electronic databases are being developed using the methodological component of the EPOC search strategy combined with selected ICT terms and free text terms relating to printed educational materials. The following are the ICT data terms that will be used in the MEDLINE search strategy. This search strategy will be translated into the other databases using the appropriate controlled vocabulary as applicable.

1. Local Area Networks/

2. exp Telemedicine/

3. (telemedicine or telehealth).tw

4. exp computer communication networks/

5. internet/

6. (ict or information communication technolog\$).tw

7. (remote communication $\$$ or remote consultation\$).tw

8. Information Services/

9. (ehealth or e-health).tw

10. (digital divide or information poverty).tw

11. (internet or email or www or world wide web or virtual or web site or website).tw

12. (e-learning or elearning or telecommunicat\$).tw

13. Databases, Bibliographic/

14. exp Medical Informatics Applications/

15. Medical Records Systems, Computerized/

16. Reminder Systems/

17. (computerised reminder $\$$ or computerised reminder $\$$ ).tw

18. Patient Identification Systems/

19. or/1-17

Any relevant references from studies found through the above routes will be followed up and obtained for assessment. International experts in the field of ICT will also be contacted to identify unpublished studies. Grey literature will be considered through Internet search engines (Google, Copernic, Alta Vista, etc.). Hand searches will be performed in specialised scientific journals with a focus on health care ICT:

- British Journal of Healthcare Computing and Information Management;

- Computer Methods and Programs in Biomedicine;

- Computers in Biology and Medicine;

- Electronic Healthcare;

- IEEE Transactions in Information Technology in Biomedicine;

- Informatics in Primary Care;

- International Journal of Electronic Healthcare;

- International Journal of Medical Informatics;

- International Journal of Healthcare Technology and Management;

- Journal of the American Medical Informatics Association;

- Journal of Medical Internet Research;

- Journal of Telemedicine and Telecare; 
- Medical Informatics and the Internet in Medicine;

- Proceedings of the AMIA Symposium;

- Telemedicine Journal and e-Health.

Finally, publications citing the selected articles will be searched through the ISI science citation index.

\section{Data collection and analysis}

Four reviewers (MPG, FL, ML, PP) will be involved in study identification and data abstraction.

To assess the influence of specific factors across studies, subgroup comparisons will be conducted to with respect to the characteristics of the technology, the intervention or the recipients. The following characteristics provide potential categories for grouping studies, but the number of subgroup comparisons will depend on the characteristics of the studies included in the review.

Characteristics of the technology:

i. Type of ICT: electronic medical record, telemedicine/telehealth, health information networks, electronic decision support tools, Internet-based technologies and services;

ii. Type of application: training, research, clinical.

Characteristics of the intervention:

i. Evidence-based: Intervention to promote ICT adoption that is evidence-based versus other intervention;

ii. Source: Intervention endorsed by official organization versus intervention from any other source;

iii. Format: Intervention in traditional format (paper based, course, lecture, self-guide) versus interventions in electronic format;

iv. Mode of delivery: Intervention delivered through mass mailing versus direct mailing versus personalised mailing;

v. Timing/frequency: Intervention given only once versus intervention given over a period of time;

vi. Complexity of behaviour: Intervention rated as low or moderate in complexity of targeted behaviour versus intervention rated as high in complexity of targeted behaviour.

Characteristics of the recipients:

i. Type of health care professional (physician, nurse, dentist, pharmacist, physiotherapist, etc.);

ii. Professional in training (excluding students) versus trained professional.

\section{Screening}

All titles and abstracts will be screened independently by two of the reviewers to assess which studies fit the inclusion criteria. Any discrepancies between the two reviewers on study inclusion will be resolved by discussion with the group of reviewers.

\section{Data abstraction}

Full text copies of all potentially relevant papers will be retrieved. Then, each study will be independently abstracted by two reviewers randomly chosen among the six members of the research team (MPG, FL, ML, PF, PP, JG). Primary authors of studies will be contacted for clarification if necessary.

\section{Quality assessment}

The quality of all eligible studies will be assessed by the two independent reviewers using the respective quality criteria applied to randomized controlled trials, controlled before and after study, and interrupted time series described in the Cochrane Effective Practice and Organisation of Care Review Group (EPOC) Data Collection Checklist (See METHODS USED IN REVIEWS under GROUP DETAILS). Any discrepancies in quality ratings will be resolved by discussion and involvement of an arbitrator where necessary.

A data extraction protocol will be used to systematically assess studies. This protocol will allow the identification of effective implementation strategies for an optimal integration of ICTs into health care professionals' practices. Any discrepancies in ratings between the two reviewers of each study will be resolved by consensus involving a third member of the team as an arbitrator. If applicable, meta-analysis will be used in order to provide a quantitative synthesis of the results.

On-going studies identified will be described, where available, detailing the primary author, research question(s), methods and outcome measures together with an estimate of the reporting date.

\section{Analytical approach}

The results for all types of comparisons will be presented using a standard method of presentation where possible. Results for categorical process outcomes, continuous process outcomes, categorical patient outcomes and continuous patient outcomes will be reported separately. For RCTs, CCTs, CBAs and ITS comparisons will be reported separately for each study design:

The number of comparisons showing a positive direction of effect; The median effect size across all comparisons;

The median effect size across comparisons without unit of analysis errors;

The number of comparisons showing statistically significant effects.

Statistical methods proposed by Grimshaw and colleagues (Grimshaw 2004a), will be used for guiding data analysis and presentation. In cases where there is insufficient data to calculate the mean effect sizes, study results will be presented in tabular form and a qualitative assessment of the effects of studies will be made, based upon the quality, the size and direction of the effect observed and the statistical significance of the studies. We will also report the range and the interquartile range of effect sizes.

For each study, data will be reported in natural units (for instance, mean number of tests ordered per physician). Where baseline results are available from RCT/CCTs, CBAs, and ITS pre-intervention and post-intervention means or proportions will be reported for both study and control groups and the absolute change from baseline will be calculated (change in study group values minus change in control group values), and where possible, with $95 \%$ confidence limits. When the baseline data is not available, results will be expressed as the relative percentage change (the difference between post-intervention values in the study and control groups expressed as a percentage of post-intervention values in the control group). Subgroups comparisons will be conducted where appropriate based upon recognised criteria (Laird 1990).

In the case of studies that present unit of analysis errors (results are reported according to a unit that differs from the allocation unit), appropriate corrections will be done, if possible, by contacting primary authors to obtain missing information. If data are not available within the correct unit of analysis, results will be reassessed and a new $p$-value will be estimated. If it is not possible 
to obtain appropriate data, only the effect size will be reported, without $p$-value.

In order to account for incorrect analysis of included ITS studies, we will reanalyse of ITS comparisons. Time series regression will be used to reanalyse each comparison (where possible). The best fit pre-intervention and post-intervention lines will be estimated using linear regression and autocorrelation adjusted for using the Cochrane-Orcutt method where appropriate (Draper 1981). First order autocorrelation will be tested for statistically using the Durbin-Watson statistic and higher order autocorrelations will be investigated using the autocorrelation and partial autocorrelation function.

\section{Effect modifiers}

The characteristics of interventions will be categorised as described below. These categories are consistent with those of other interventions aimed at changing health care professional practices (Burgers 2003; Foy 2002; Grilli 1994; Grol 1998; Kanouse 1995; Kreuter 1996; Marriott 2000; Wathen 2002).

Characteristics which may influence the effectiveness of interventions to promote ICT adoption/use in health care settings are:
(1) Type of technology: electronic medical record, telemedicine/ telehealth, health information networks, decision support tools, Internet-based technologies and services;

(2) Potential adopter: type of health care professionals;

(3) Intervention:

(a) Source: endorsed by official organisation, evidence-based;

(b) Format: written information, lecture/course/tutorial, followup/feedback, etc.;

(c) Mode of delivery: mass delivery, personalised delivery, face to face, electronic, other;

(d) Content of the intervention: ICT knowledge and skills, clinical compliance with a standard or guideline, information about costs, other; tailored; theory-based.

We will visually explore the heterogeneity by preparing tables and bubble plots that group comparisons relative to each of these variables in relationship to the size of the effect. The rational for pooling different types of interventions is to determine the impact of heterogeneity among interventions rather than to estimate the effects of a homogenous intervention, as is usually the case for a meta-analysis (Kawamoto 2005). 


\section{REFERE N C E S}

\section{Additional references}

\section{Aarts 2004}

Aarts J, Doorewaard H, Berg M. Understanding implementation: The case of a computerized physician order entry system in a large Dutch university medical center. Journal of American Medical Informatics Association 2004;11(3):207-16.

\section{Allen $\mathbf{2 0 0 0}$}

Allen M, Kaufman D, Barrett A, Paterson G, Sargent J, Mcleod R. Self-Reported Effects of Computer Workshops on Physician's Computer Use. Journal of Continuing Education for Health Professionals 2000;20:20-6.

\section{Bennett 2003}

Bennett JW, Glasziou PP. Computerised reminders and feedback in medication management: a systematic review of randomised controlled trials. The Medical Journal of Australia 2003;178(5):217-22.

\section{Berner 2005}

Berner ES, Detmer DE, Simborg D. Will the Wave Finally Break? A Brief View of the Adoption of Electronic Medical Records in the United States. Journal of American Medical Informatics Association 2005;12(1):3-7.

\section{Brooks 2006}

Brooks RG, Menachemi N. Physicians' Use of Email with Patients: Factors Influencing Electronic Communication and Adherence to Best Practices. Journal of Medical Internet Research 2006;8(1):e2.

\section{Bull 2001}

Bull F, Kreuter M, Clark E, Scharff D. Understanding the effects of printed health education material which features lead to which outcomes. Journal of Health Communication 2001;6(3):265-79.

\section{Burgers 2003}

Burgers J, Grol R, Zaat J, Spies T, van der Bij A, Mokkink H. Characteristics of effective guidelines for general practice. British Journal of General Practice 2003;53(486):15-9.

\section{Carman 1996}

Carman J, Shortell S, Foster R, Hughes E, Boerstler H, O'Connor EJ, et al. Keys for Successful Implementation of TQM in Hospitals. Health Care Management Review 1996;2(1):48-60.

\section{Currell 2000}

Currell R, Urquhart C, Wainwright P, Lewis R. Telemedicine versus face to face patient care: effects on professional practice and health care outcomes (Cochrane Review). The Cochrane Database of Systematic Reviews 2000, Issue 2. [Art. No.: CD002098. DOI: 10.1002/14651858.CD002098]

\section{Davis 1999}

Davis D, O'Brien MA, Freemantle N, Wolf FM, Mazmanian P, Vaisey-Taylor A. Impact of formal continuing education: Do conferences, workshops, rounds, and other traditional continuing education activities change physician behaviour or health care outcomes ?. JAMA 1999;282(9):867-74.

\section{Draper 1981}

Draper NR, Smith H. Applied Regression Analysis. New York: Wiley and Sons, 1981:709 p..

\section{Erstad 2003}

Erstad TL. Analyzing computer based patient records: a review of literature. Journal of Healthcare Information Management 2003;17(4):51-7.

\section{Eysenbach 2001}

Eysenbach G. What is e-health ?. Journal of Medical Internet Research 2001;3(2):e20.

\section{Foy 2002}

Foy R, McLennan G, Grimshaw J, Penney G, Campbell M, Grol R. Attributes of clinical recommendations that influcence change in practice following audit and feedback. Journal of Clinical Epidemiology 2002;55:717-22.

\section{Gagnon 2005}

Gagnon M-P, Lamothe L, Fortin J-P, Godin G, C Gagné, reinharz $\mathrm{D}$, et al. Telehealth adoption in hospitals: An organisational perspective. Journal of Health Organization and Management 2005;19(1):32-56.

\section{Garg 2005}

Garg AX, Adhikari NKJ, McDonald H, Rosas-Arellano MP, Devereaux PJ, Beyene J, et al. Effects of computerized clinical decision support systems on practitioner performance and patient outcomes. A systematic review. JAMA 2005;293:1223-38.

\section{Gov. Canada 1999}

Governement of Canada. Federal, Provincial and Territorial Advisory Committee on Population Health. Towards a healthy future. Second report on the health of Canadians. Ottawa 1999.

\section{Grilli 1994}

Grilli R, Lomas J. Evaluating the message: The relationship between compliance rate and the subject of a practice guideline. Medical Care 1994;32:202-13.

\section{Grimshaw 2004}

Grimshaw JM, Eccles MP. Is evidence-based implementation of evidence-based care possible?. Medical Journal of Australia 2004;180(6 Suppl):S50-1.

\section{Grimshaw 2004a}

Grimshaw JM, Thomas RE, MacLennan G, Fraser C, Ramsay CR, Vale L, Whitty P, Eccles MP, Matowe L, Shirran L, Wensing M, Dijkstra R, Donaldson C. Effectiveness and efficiency of guideline dissemination and implementation strategies. Health Technology Assessment 2004;8(6):iii-72. 


\section{Grol 1998}

Grol R, Dalhuijsen J, Thomas S, Veld C, Rutten G, Mokkink H. Attributes of clinical guidelines that influence of guidelines in general practice: Observational study. BMJ 1998;317:858-61.

\section{Grol 2004}

Grol R, Wensing M. What drives change ? Barriers to and incentives for achieving evidence-based practice. Medical Journal of Australia 2004;180(6 Suppl):S57-60.

\section{Health Canada 2006}

Health Canada. eHealth Thesaurus. http://www.hc-sc.gc.ca/hcssss/ehealth-esante/res/thesaurus/index_e.html (last checked 03-27-2006).

\section{Kanouse 1995}

Kanouse D, Kallich J, Kahan J. Dissemination of effectiveness and outcomes research. Health Policy 1995;34:167-192.

\section{Kawamoto 2005}

Kawamoto K, Houlihan CA, Balas EA, Lobach DF. Improving clinical practice using clinical decision support system: a systematic review of trials to identify features critical to success. BMJ 2005;330(7494):765.

\section{Kimberly 1981}

Kimberly JR, Evanisko MJ. Organizational innovation: The influence of individual, organizational and contextual factors on hospital adoption of technological and administrative innovations. Academy of Management Journal 1981;24(4):689-713.

\section{Knonick 2003}

Kronick J, Blake C, Munoz E, Heilbrunn L, Dunikowski L, Milne WK. Improving on-line skills and knowledge. A randomized trial of teaching rural physicians to use on-line medical information. Canadian Family Physician. 2003;49:312-7.

\section{Kreuter 1996}

Kreuter MW, Strecher VJ. Do tailored behaviour change messages enhance the effectiveness of health risk appraisals? Results from a randomized controlled trial. Health Education Research 1996;11(1):97-105.

\section{Kreuter 2001}

Kreuter M, Holt C. How do people process health information ? Applications in an age of individualized communication. Current Directions in Psychological Science. 2001;10(6):206-9.

\section{Laird 1990}

Laird N, Mosteller F. Some statistical methods for combining experimental results. International Journal of Technology Assessment in Health Care 1990;6(5):30.

\section{Lapointe 1999}

Lapointe L, Rivard S. [L'implantation d'un système d'information clinique]. ASAC Conference, St-John (N.-B.).. 1999.

\section{Lorenzi 1997}

Lorenzi NM, Riley RT, Blyth AJ, Southon G, Dixon BJ. Antecedents of the people and organizational aspects of medical informatics: Reviews of the literature. Journal of American Medical Informatics Association 1997;4(2):79-83.

\section{Markus 1983}

Markus ML. Power, politics and MIS implementation. Communications of the ACM. 1983;26(6):430-44.

\section{Marriott 2000}

Marriott S, Palmer C, Lelliott P. Disseminating healthcare information: Getting the message across. Quality in Health Care 2000;9:58-62.

\section{Massaro 1993}

Massaro T. Introducing physician order entry at a major academic medical center: I. Impact on organizational culture and behavior. Academic Medicine 1993;68(1):20-5.

\section{McLaughlin 1998}

McLaughlin J, Webster A. Rationalising knowledge: IT systems, professional identities and power. Sociological Review. 1998;46:781-802.

\section{Moehr 2000}

Moehr J, Grant A. Medical Informatics and Medical Education in Canada in the 21st Century. Clinical and Investigative Medicine 2000;23(4):275-280.

\section{Oh 2005}

Oh H, Rizo C, Enkin M, Jadad A. What Is eHealth (3): A Systematic Review of Published Definitions. Journal of the Medical Internet Research. 2005;7(1):e1.

\section{Open Clinical 2006}

Open Clinical. E-Health. http://www.openclinical.org/eHealth.html (last checked 03-27-2006).

\section{Pluye 2005}

Pluye P, Grad R, Dunikowski LG, Stephenson R. The impact of clinical information-retrieval technology on physicians: A literature review of quantitative, qualitative and mixedmethod studies. International Journal of Medical Informatics 2005;74:745-68.

\section{Ramsay 2003}

Ramsay CR, Grilli R, Thomas RE, Matowe L, Grimshaw JM. Interrupted Time Series Designs in Health Technology Assessment: Lessons from Two Systematic Reviews of Behavior Change Strategies. International Journal of Technology Assessment in Health Care 2003;19(4):613-23.

\section{Slotnick 1999}

Slotnick H. How doctors learn: physicians self-directed learning episodes. Academic Medicine 1999;74(10):1106-17.

\section{Street 1997}

Street R, Rimal R. Health promotion and interactive technology: A conceptual foundation. In: Street R, Gold W, Manning T editor(s). Health promotion and interactive technology: 
Theoretical applications and future directions. New Jersey: Lawrence Erlbaum Associates, 1997:19-38.

\section{Tseng 1999}

Tseng S, Fogg B. Credibility and computing technology.

Commun ACM. 1999;42(5):39-44.

\section{WHAT'S NEW}

\section{Wathen 2002}

Wathen CN, Burkell J. Believe it or not: Factors influencing credibility on the web. Journal of the American Society for Information Science and Technology. 2002;3(2):134-44.

\begin{tabular}{lll}
\hline Date & Event & Description \\
\hline 14 August 2008 & Amended & Converted to new review format. \\
\hline
\end{tabular}

\section{CONTRIBUTIONSOF AUTHORS}

All review authors have contributed to the production of the protocol. MPG lead the writing of the protocol, and FL, ML, PP, PF and JG provided comments and feedback. For the full review: KG will develop and run the search strategy. MPG and KG will screen records for eligibility. FL will act as arbitrator should disagreement arise. MPG, KG, FL, ML will abstract data, undertake analysis, interpret the results and write up the review. PP, PF, and JG will be consulted through the review process to provide feedback and comments.

\section{DECLARATIONSOF INTEREST}

No conflict of interest identified.

\section{N DEX TERMS}

\section{Medical Subject Headings (MeSH)}

Databases, Bibliographic [utilization]; Health Personnel [ ${ }^{*}$ statistics \& numerical data]; Information Storage and Retrieval [ ${ }^{*}$ utilization]; Professional Practice [ ${ }^{\star}$ statistics \& numerical data]; Randomized Controlled Trials as Topic 